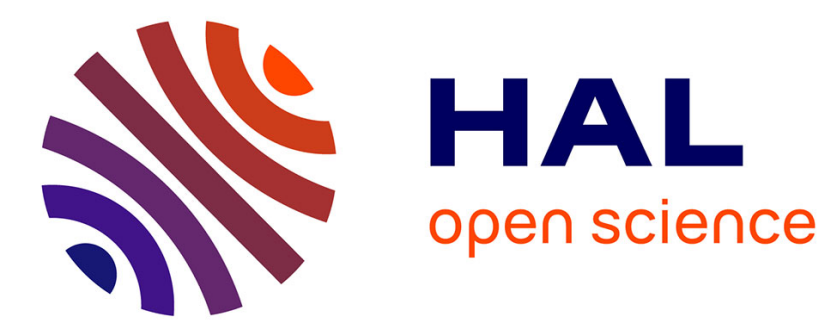

\title{
Cooperation in two-person evolutionary games with complex personality profiles
}

\author{
Tadeusz Platkowski
}

\section{To cite this version:}

Tadeusz Platkowski. Cooperation in two-person evolutionary games with complex personality profiles. Journal of Theoretical Biology, 2010, 266 (4), pp.522. 10.1016/j.jtbi.2010.07.018 . hal-00620583

\section{HAL Id: hal-00620583 https://hal.science/hal-00620583}

Submitted on 8 Sep 2011

HAL is a multi-disciplinary open access archive for the deposit and dissemination of scientific research documents, whether they are published or not. The documents may come from teaching and research institutions in France or abroad, or from public or private research centers.
L'archive ouverte pluridisciplinaire HAL, est destinée au dépôt et à la diffusion de documents scientifiques de niveau recherche, publiés ou non, émanant des établissements d'enseignement et de recherche français ou étrangers, des laboratoires publics ou privés. 


\section{Author's Accepted Manuscript}

Cooperation in two-person evolutionary games with complex personality profiles

Tadeusz Płatkowski

PII: S0022-5193(10)00375-9

DOI: doi:10.1016/j.jtbi.2010.07.018

Reference: YJTBI 6080

To appear in: Journal of Theoretical Biology

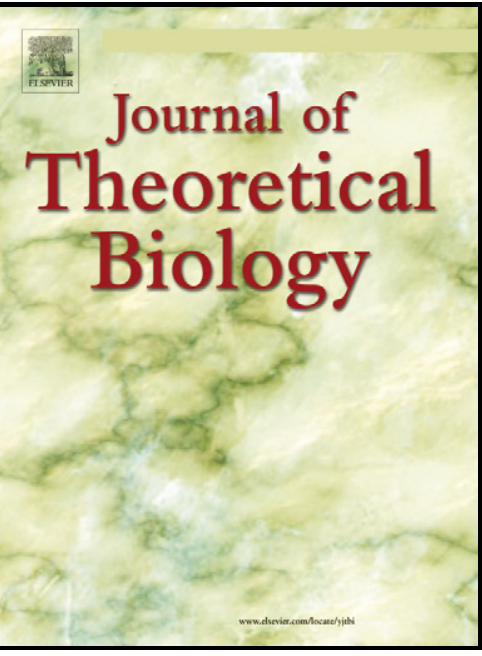

www.elsevier.com/locate/yjtb

Received date: 25 January 2010

Revised date: $\quad 19$ July 2010

Accepted date: 19 July 2010

Cite this article as: Tadeusz Płatkowski, Cooperation in two-person evolutionary games with complex personality profiles, Journal of Theoretical Biology, doi:10.1016/j.jtbi.2010.07.018

This is a PDF file of an unedited manuscript that has been accepted for publication. As a service to our customers we are providing this early version of the manuscript. The manuscript will undergo copyediting, typesetting, and review of the resulting galley proof before it is published in its final citable form. Please note that during the production process errors may be discovered which could affect the content, and all legal disclaimers that apply to the journal pertain. 


\author{
Tadeusz Płatkowski* \\ Department of Mathematics, Informatics and Mechanics, \\ University of Warsaw, Banacha 2, 02-097 Warsaw, Poland
}

\begin{abstract}
We propose a theory of evolution of social systems which generalizes the standard proportional fitness rule of the evolutionary game theory. The formalism is applied to describe the dynamics of two-person one-shot population games. In particular it predicts the non-zero level of cooperation in the long run for the Prisoner's Dilemma games, the increase of the fraction of cooperators for general classes of the Snow - Drift game, and stable nonzero cooperation level for coordination games.
\end{abstract}

PACS numbers:

Keywords: Population Dynamics, Prisoner's Dilemma, Mixed Equilibria

\title{
I. INTRODUCTION
}

Game theory has been proved to be an effective tools of modeling different types of interactions in various scientific disciplines, e.g. in biology, social sciences and economics, cf. e.g. Vega-Redondo (2003), Nowak (2006), Szabo and Fath (2007), McElreath and Boyd (2007), Gintis (2009) and references therein. The usual assumptions of the perfect rationality lead to results which contradict many real and laboratory experimental situations. The standard game theory as a language of modeling the social interactions adopts too strong assumptions concerning individual behaviors, its motivations and regulatory mechanisms.

We propose a theory of evolution of social systems which generalizes the standard proportional fitness rule of the evolutionary game theory. The biological fitness of a behavior, strategy, measured by the payoff from interactions, is replaced by more general function, the attractiveness of the behavior. The attractiveness of the strategy is assumed to depend not only on its payoff, but also on its actual popularity (fraction) in the population. The parameters of the attractiveness function describe different psychological characters of the agents.

We formulate the evolution equation for the two-persons symmetric games with two strategies and find the equilibria and determine their stabilities for the considered mean field type population models. For such games we prove the existence of a polymorphic stable equilibrium. We determine sufficient conditions for which the equilibrium is unique. We also discuss the generic games with three mixed equilibria, two of them stable. Their existence is important in particular for the temporal evolution - the mixed equilibrium time asymptotic state depends for such games not only on the personality profiles of the actors and the payoff matrix of the game, but also on the initial composition (i.e. the fractions of the agents who play each available strategy) of the population. For the games with unique mixed equilibrium we study the dependence of the equilibrium composition of the strategies on the personality profiles of the actors.

The PD game is one of the most popular and important games in social sciences, studied as a paradigm for the evolution of cooperation. In the models of continuous systems of actors playing at each instant of time the one-stage PD, e.g. in the mean field type equations of the replicator dynamics, the only asymptotic equilibrium state is defection. There are various 'solutions' of the dilemma, aimed at maintaining cooperation in the systems of agents in the long run. One can add spatial structure in the model, consider repeated interactions, learning through the introduction of the aspiration levels etc. Our model provides another solution of the dilemma, allowing - for general psychological profiles - for the persistence of cooperation in the long run. The theory is applied to fairly general two-person games, including the Snow-Drift game and the coordination games.

\section{MODEL}

We consider the evolutionary scenario: infinite homogeneous population of individuals. The players interact through a random pairwise matching, playing at each instant of time a 2- person symmetric game with two behavioral types

*Electronic address: tplatk@mimuw.edu.pl 


\section{\begin{tabular}{l|ll} 
& $\mathrm{C}$ & $\mathrm{D}$ \\
\hline $\mathrm{C}$ & $\mathrm{R}$ & $\mathrm{S}$
\end{tabular} \\ $\mathrm{D} \mid \mathrm{T} \mathrm{P}$}

abbreviated in the following by $[R, S, T, P]$, where $R, S, T, P$ are arbitrary positive numbers.

In general $\mathrm{C}$ and $\mathrm{D}$ denote any two strategies. The most important applications will cover the social dilemmas, in particular the Prisoner's Dilemma game (PD), and for the Snow Drift game (SD), for which C stands for cooperation, $\mathrm{D}$ for defection, $\mathrm{R}$ for reward, $\mathrm{S}$ for sucker, $\mathrm{T}$ for temptation, and $\mathrm{P}$ for punishment. Another applications refer to the coordination games, for which we keep the same notation, cf. next section.

We denote: $p_{i}=p_{i}(t), i=1,2$ : the fraction of the strategy $i$ (popularity, propensity of $i$ ) in the population at time $t$, $\nu_{1}=R p_{1}+S p_{2}, \nu_{2}=T p_{1}+P p_{2}$ : the mean payoffs of the strategy respectively $\mathrm{C}$ and $\mathrm{D}$ in the population,

$u_{i}(t)$ : the attractiveness of the strategy $i$ at time t.

The attractiveness of the strategy $i$ in the population reflects the fact that more attractive strategies ought to have an evolutionary advantage in the considered social systems. The attractiveness of the strategy $i$ will be assumed to be an increasing and concave function of its mean payoff $\nu_{i}$ and the popularity $p_{i}$ in the population. The concavity reflects the fact that when the attractiveness reaches a higher level, the changes are slower. It is also expected that the attractiveness of the strategy becomes zero if its mean payoff or its popularity in the population is zero. In our model we choose for the attractiveness of the strategy $i$ the Cobb-Douglas utility function, cf. Cobb and Douglas (1928), Platkowski and Poleszczuk (2009):

$$
u_{i}(t)=p_{i}^{1-\alpha} \nu_{i}^{1-\beta}, \quad i=1,2,
$$

with $(\alpha, \beta) \in[0,1] \times[0,1]$.

The formula (1) states that the attractiveness of a strategy depends not only on the payoff of the strategy, but also on its actual popularity in the population.

The parameters $\alpha, \beta$ determine the responsiveness of the function $u_{i}$ to changes of the actual values of the current popularity and of the mean payoff of the action $i$. They define diverse personality profiles of the individuals.

Exploiting Weber's intuitions, cf. Weber (1968), we defined the ideal types of the personality profiles for the limiting values of the parameters $\alpha, \beta$, and characterized their basic properties, cf. Platkowski and Poleszczuk (2009):

HE (Homo Economicus): $\alpha=1, \beta=0$. It means that $\mathrm{HE}$ assesses attractiveness of the action exclusively through its effectiveness $\left(u_{i}=\nu_{i}\right)$. HE is interested exclusively in the future prospects, In the Weber's typology this sort of actor's motivation is called Zweckrationalitat (future-oriented instrumental rationality).

HS (Homo Sociologicus): $\alpha=0, \beta=1$. The HS assesses attractiveness of the action only through its popularity, the past effect for him/her $\left(u_{i}=p_{i}\right)$. HS is insensitive to the payoffs of the game.

HT (Homo Transcendentalis): $\alpha=\beta=1$. It is an ideal type, for which every action has the same attractiveness $\left(u_{i}=1\right)$. It describes a personality not interested in the effectiveness of a behavior or in its propensity, but rather by some other values. Thus, HT is the ideal type insensitive to the payoffs and the popularities of the strategies. This type of actor's orientation is called Wertrationalitat in Weberian terms.

HA (Homo Afectualis): $\alpha=\beta=0$. The attractiveness function takes the form $u_{i}=\nu_{i} p_{i}$ The corresponding evolution equations reduce to the standard replicator equations, as will be shown below.

All the other values of the parameters $\alpha, \beta$ describe intermediate, mixed personality profiles. We show below that the relevant combination of these parameters, defined through the sensitivity parameter $s=\frac{1-\beta}{\alpha}$ plays a crucial role in determining the temporal evolution of the considered populations. At the end of this section we discuss the sensitivities of the ideal personality profiles defined above.

The notion of attractiveness generalizes the notion of fitness in the classical evolutionary game theory, in which a linear relation between payoff and fitness is usually assumed, mainly for its mathematical advantages. In general the relation (1) gives nonlinear dependence of the attractiveness on the payoffs. We note that e.g. the exponential dependence of the fitness on payoffs has been considered in Traulsen et al. (2008); Aviles (1999). The reader is also referred to Henrich and Boyd (2001) for another approach in which the ideas of imitating the successful, and copying the majority (the conformist transmission), are put together to stabilize the cooperation in populations of individuals.

Many social and biological interactions are based on imitation processes, where individuals are more likely to adopt more successful and more popular strategies. We assume that the change of the distribution of the strategies in the system occurs through the process of imitation of the more attractive strategies.

Let $K$ be the number of available strategies (in this paper $K=2$ ). Let $N_{i}(t)$ denotes the number of individuals playing the action $i, N=N_{1}+N_{2}+\ldots N_{K}$ - the fixed size of the system, $p_{i}=N_{i} / N$ - the propensity of the behavior $i$ for $i=1,2, \ldots, K$. 
Assuming that the $i$-strategists review their strategy according to the Poisson process with the arrival rate $r_{i}$ we model the corresponding stochastic processes as a deterministic flow. The balance conservation equations read, cf. Weibull (1995), Section 4.4:

$$
\dot{p}_{i}(t)=\sum_{j \neq i}\left[p_{j} r_{j} p_{j}^{i}-p_{i} r_{i} p_{i}^{j}\right], \quad i=1, \ldots K
$$

where $p_{j}^{i}$ is the probability that the agent playing the $j$ strategy will switch to the $i$ strategy.

We assume that $p_{j}^{i}$ is proportional to the attractiveness $u_{i}$ of the strategy $i$ :

$$
p_{j}^{i}=c u_{i}
$$

$c$ being the same for all strategies: the strategies with higher attractivenesses have larger probability to be imitated. Assuming that the arrival rates $r_{j}$ are state and strategy independent constants, we obtain, after a straightforward calculation and time rescaling

$$
\dot{p}_{i}(t)=u\left(\frac{u_{i}}{u}-p_{i}\right), \quad u:=\sum_{j=1}^{K} u_{j}, \quad i=1,2, \ldots, K .
$$

The evolution equations (4) state that the change of $p_{i}$ is governed by its relation to the reference function - the normalized attractiveness $\tilde{u}_{i}:=\frac{u_{i}}{u}$, and that the fraction $p_{i}$ of the strategy $i$ increases if $\tilde{u}_{i}$ is bigger than the actual fraction of the strategy $i$ in the population, and decreases if it is smaller.

We study the time evolution and the fixed points (equilibria) of the dynamical system (4) for $K=2$ strategies. With the normalization condition $p_{1}+p_{2}=1$ eq. (4) reduces to the evolution equation

$$
\dot{p}_{1}=\left(1-p_{1}\right)^{1-\alpha} p_{1}^{1-\alpha}\left[\nu_{1}^{1-\beta}\left(1-p_{1}\right)^{\alpha}-\nu_{2}^{1-\beta} p_{1}^{\alpha}\right],
$$

with $\nu_{1}=(R-S) p_{1}+S, \quad \nu_{2}=(T-P) p_{1}+P$.

We note that for each personality profile $(\alpha, \beta) \in[0,1] \times[0,1]$ there exist two pure equilibria of $(4): p_{1}=0$ and $p_{1}=1$ [for $\alpha=1$ we put $\left.u_{i}\left(p_{i}=0\right)=0, i=1,2\right]$. All the other equilibria of eq. (4) will be called Mixed Equilibria, and denoted ME. Thus, each ME corresponds to a fixed point of eq. (5).

We find a simple transformation

$$
z=\frac{p_{1}}{1-p_{1}}, \quad p_{1} \in(0,1)
$$

which allows to study important mathematical properties of ME in our model. In Appendix A (cf. Proposition 1) we show that the stationary solutions of $(5)$ in the interval $(0,1)$, as well as their stability properties are the same as those of the equation

$$
\dot{z}=W(z) \equiv\left[\frac{R z+S}{T z+P}\right]^{s}-z, \quad z \in(0, \infty)
$$

where we introduced the sensitivity to reinforcements parameter $s$ :

$$
s=\frac{1-\beta}{\alpha}, \quad \alpha \neq 0 .
$$

Formula (8) implies that the existence and the asymptotic properties of the solutions of eq. (4) depend on the combination of the parameters $\alpha, \beta$, which characterize the personality profile of the players, rather than separately on each of them. The sensitivity parameter $s$ plays an important role in the matching law in the operant response theory of the mathematical psychology, cf. Hernstein (1997), Seth (2002), Platkowski and Poleszczuk (2009), and references cited therein. To see the connection we derive from (4) the equilibrium condition (for $K=2$ ):

$$
\frac{u_{1}}{p_{1}}=\frac{u_{2}}{p_{2}},
$$

which, after substituting (1) and (8) is equivalent to the matching law of the mathematical psychology:

$$
\frac{p_{2}}{p_{1}}=\left(\frac{\nu_{2}}{\nu_{1}}\right)^{s} .
$$


The parameter $s$ is a measure of the degree to which, in equilibrium, the response ratio changes when the reinforcement ratio is modified.

In particular, the weberian ideal type Homo Transcendentalis: $\alpha=\beta=1$, for which $s=0$, describes the personalities insensitive to reinforcements. The ideal type Homo Economicus: $\alpha=1, \beta=0$ corresponds to the sensitivity $s=1$. Note that in the Cobb-Douglas utility function (1) the case $\alpha+\beta=1$ is referred to as the constant return to scale in the mathematical economics. This case corresponds also to the third ideal type, Homo Sociologicus: $\alpha=0, \beta=1$, for which every state vector $\left(p_{1}, p_{2}\right)$ is an equilibrium, as can be seen from (5). Finally the "opposite" to Homo Transcendentalis ideal type, Homo Afectualis, $\alpha=\beta=0$, corresponds to infinite sensitivity. In this case the evolution equations (4) are identical to the replicator equations in the evolutionary game theory. To see it note that for $\alpha=0$ eqs. (4) can be rewritten in the form

$$
\dot{p}_{i}=p_{i} \sum_{j=1, \ldots K} p_{j}\left[\nu_{i}^{1-\beta}-\nu_{j}^{1-\beta}\right], \quad i=1, \ldots K .
$$

In particular for $\beta=0$ we obtain the usual replicator equations for the two-person symmetric games with $K$ strategies. For $K=2$ strategies we obtain from eqs. (11) the modified replicator dynamics

$$
\dot{p}_{1}=p_{1}\left(1-p_{1}\right)\left[\nu_{1}^{1-\beta}-\nu_{2}^{1-\beta}\right] .
$$

In the next section we discuss the existence, uniqueness and stability properties of the equilibrium states of our model. Results are proved for general payoff matrices of the two-person symmetric games, including the most popular two-person social dilemmas: the Prisoner's Dilemma and the Snow -Drift games as well as the general Coordination games.

\section{EQUILIBRIA}

\section{A. General results}

Let $p^{*} \in(0,1)$ denotes a Mixed Equilibrium (ME), i.e. a fixed point of eq. (5). Thus, $p^{*}$ is the fraction of the individuals who play the first (C) strategy in equilibrium. When discussing the social dilemmas, with $\mathrm{C}$ denoting the first, cooperation strategy, the value $p^{*}$ will be called the (asymptotic) cooperation level in the population.

The inverse transformation $z^{*}:=\frac{p^{*}}{1-p^{*}}$, cf. (6) allows to identify ME with the zeros $z^{*}$ of the function $W(z)$ in $(7)$ : $W\left(z^{*}\right)=0$.

We study ME and their stability under the dynamics (5), or equivalently (7). In particular, for social dilemmas described by the relevant two - person games with cooperation and defection as two available strategies (e.g. for the $\mathrm{PD}$ or the SD game) the stable ME describe the states for which the partial cooperation is an asymptotic outcome of the considered social systems.

We prove the following theorem on the existence, uniqueness and stability of ME for the considered two-person symmetric games:

\section{Theorem 1}

For the payoff matrix $[R, S, T, P]$ with positive entries:

I. For each $0 \leq s<\infty$ there exists at least one $M E$ - the fixed point of the evolution equation (5).

II. Denote $B:=(1-s) \frac{P}{T}+(1+s) \frac{S}{R}, \Delta:=B^{2}-4 \frac{S P}{R T}$. If $1 . \Delta \leq 0$, or $2 . B \geq 0$, or $3 . S T \geq R P$, then ME is unique.

III. For each $0 \leq s<\infty$ there exist at most three $M E$.

IV. There exist three $M E$ iff $\Delta>0, \quad B<0$, and $U\left(z_{1}\right) U\left(z_{2}\right)<0$, where $z_{1,2}:=[-B \mp \sqrt{\Delta}] / 2$, and $U(z):=$ $\ln z+s \ln \frac{T z+P}{R z+S}, \quad z>0$.

$\mathrm{V}$. If $M E$ is unique then it is globally stable in $(0,1)$ under the dynamics (5). If there are three $M E$, then the smallest and the largest ME are stable, the middle one is unstable.

Proof:

I. Taking logarithm of eq. $W(z)=0$, cf. (7), we obtain 1-1 correspondence between the zeros of the function $U(z)$ defined in Theorem 1, and the ME - the solutions of (7). The proof follows by noting that for positive entries of the payoff matrix $[R, S, T, P]$ we have $\lim _{z \rightarrow 0} U(z)=-\infty, \quad \lim _{z \rightarrow+\infty} U(z)=+\infty$.

P. II-IV are proved by studying the extrema of $U(z)$ (details are given in Appendix A). 
P. V follows from the observation that in eqs (5) for all $\alpha_{1} \in(0,1], \beta \in[0,1]$ we have $\dot{p}_{1}>0$ for $p_{1}>0$ small enough, and $\dot{p}_{1}<0$ for $1-p_{1}$ small enough (whereas the expression in the square brackets in (5) is positive for $p_{1}$ small enough, and negative for $1-p_{1}$ small enough).

The statement I implies that the dependence of the utility function (attractiveness) of a strategy on the propensity of the strategy results in the existence of at least one ME for all symmetric two-person games with positive payoffs, including all types of the social dilemmas for such games. In particular this "solves the dilemma" of the Prisoner's Dilemma game in the considered populations.

An application of the sufficient conditions II is given in Example 3, Appendix B. In particular, p. II.2 implies that for $s \leq 1$ the ME is unique. P. V states its global stability. Note that for $s \leq 1$ the cooperation level does not depend on the initial distribution of the strategies in the population. On the other hand, if the sufficient conditions of p.IV are satisfied, then there are two (locally) stable fixed points of eq. (5), and the cooperation level does depend on the initial state of the population.

P. II.3 provides the sufficient condition for the uniqueness of ME for all $s \geq 0$. It states that the the ME is unique if the product of payoffs from playing different strategies is bigger than that from playing the same strategies, e.g. for the class of the anti-coordination games considered below.

P. V implies that if the ME is unique, then the pure equilibria, which correspond to the fixed points $p_{1}=0$ and $p_{1}=1$ of eq. (4) are unstable, the polymorphic asymptotic state of the population is the only stable one. This implies for example that in such cases the monomorphic equilibria (all defectors or all cooperators) are unstable for the social dilemmas games.

Examples and applications of Theorem 1 to the two-person social dilemmas will be discussed in the next subsections. For completeness, in Appendix B we provide analogous existence theorems for the payoff matrices which admit zero entries.

\section{B. Equilibria for PD Games}

The PD game is a two-person symmetric game with the payoff matrix the entries of which are denoted

$$
[R, S, T, P], \quad T>R>P>S
$$

where $R$ (Reward), and $S$ (Sucker) are the payoffs for $\mathrm{C}=$ Cooperation when the opponent plays respectively C, and $\mathrm{D}$, whereas $T$ (Temptation), and $P$ (Punishment) are the payoffs for $\mathrm{D}=$ Defection when the opponent plays respectively $\mathrm{C}$, and D.

First we discuss the dependence of the cooperation level $p^{*}$ on the payoffs and on the sensitivity parameter $s$ for such PD games, for which Theorem 1 guarantees the uniqueness of ME. As expected, $p^{*}$ increases for increasing $R$ and decreasing $T, P$, cf. Fig. 1 for different PD payoff matrices. Moreover, $p^{*} \rightarrow 0$ when $s \rightarrow+\infty$, in agreement with the previously discussed fact that our dynamics approaches the modified replicator dynamics (12) in this limit.

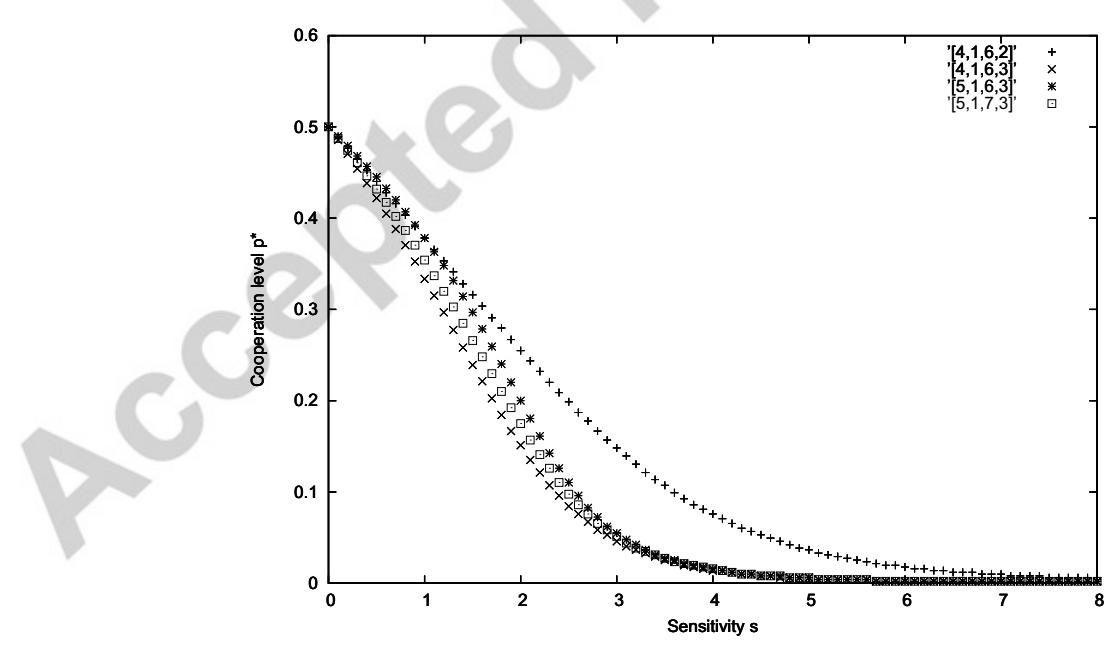

FIG. 1: Cooperation level vs. sensitivity for various PD games. The components of the vectors in the legend correspond to the entries of the payoff matrix $[R, S, T, P]$. 
In general the uniqueness of the ME does not hold for all types of the PD games (13). We found some particular types of PD (i.e. rather 'narrow' intervals of the entries of the general PD matrix), for which there exist three ME. To this end we performed calculations for all integer entries of the payoff matrix (13) from the set $\{1, \ldots .40\}$, checking the sufficient conditions for the existence of three ME formulated in Theorem 1, p. V for various sensitivities $s$. For example for the sensitivity $s=2$ there are only five such integer valued matrices: $[R, S, T, P]=[33,1,36,10],[32,1,33,10],[37,1,38,11]$, $[38,1,40,11],[37,1,39,11]$. In Fig. 2 we show the cooperation levels as functions of the sensitivity $s$ for the PD game $[R, 1,30,5]$ for different values of the reward $R$. For clarity we plotted discretized values, the full plots are continuous. Note the existence of three ME for a "narrow" interval of sensitivities around $s=3$ for $R=27,29$; one can easily checked that the sufficient conditions for their existence, formulated in p. IV, Theorem 1, are satisfied.

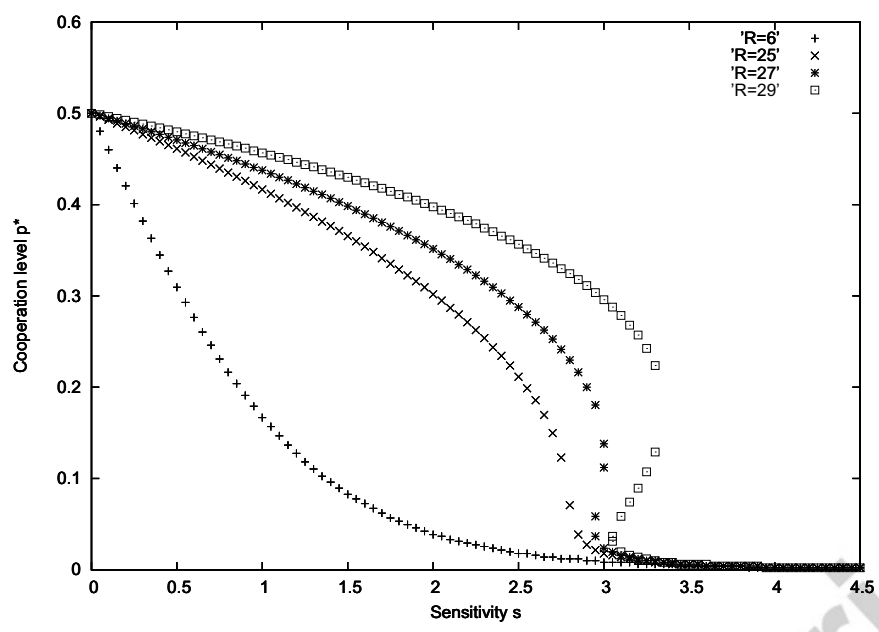

FIG. 2: Cooperation levels vs. sensitivity for increasing reward $R$ for the $\mathrm{PD}$ game $[R, 1,30,5]$. For $\mathrm{R}=6$ and $\mathrm{R}=25$ the $\mathrm{ME}$ is unique. For $\mathrm{R}=27$ and $\mathrm{R}=29$ there are three $\mathrm{ME}$ in the narrow interval of $s$ around $s=3$. For example for $\mathrm{R}=29$ and the sensitivity $s=3.2$ two stable ME correspond to the cooperation levels $p^{*} \approx 0.25$ and $p^{*} \approx 0.02$, whereas the unstable ME corresponds to $p^{*} \approx 0.08$.

\section{Equilibria for Anti-coordination Games}

We define the general Anti-coordination game with the payoff matrix

$$
[R, S, T, P], \quad T>R>0, \quad S>P \geq 0 .
$$

The game has one mixed and two pure Nash equilibria, the mixed one stable under the replicator dynamics. We prove

\section{Lemma 1}

For all sensitivities $s \in[0,+\infty)$ there exists an unique $M E$ for the Anti-coordination game (14) - a global attractor of the dynamics (5).

Proof: Using the notation of Theorem 1 we calculate

$$
B=s(S T-R P) / R T+P / T+S / R .
$$

For the payoff matrix (14) $B>0$, therefore the uniqueness and the global attraction property of the ME follows respectively from p. II.2 and $\mathrm{V}$ of Theorem 1.

Note that this result holds for $P \geq 0$, i.e. in particular for the - important for applications in social sciences - the Snow-Drift (SD) game, cf. Sugden (1986), Hauert and Doebeli (2004).

Let us consider the SD game with the payoff matrix in the (b)enefit - (c)ost formulation:

$$
[b-c / 2, b-c, b, 0], \quad b>c>0 .
$$

This game represents the social dilemma in which the strategy $\mathrm{C}$ - cooperation implies a benefit $b$ to the cooperator and to the opponent (independently of the opponent's strategy), and in addition incurs the cost $c$ if the opponent plays the strategy $\mathrm{D}$ (i.e. defects), or $c / 2$ if (s)he cooperates. If both players defect, they receive 0. 
In the mixed Nash equilibrium of the replicator dynamics the fraction of $\mathrm{C}$ - players is equal $p_{N}=2(b-c) /(2 b-c)$. In Fig. 3 we plot the cooperation level $p^{*}$ in function of the cost $c$ for the normalized $(b=1)$ SD game with the payoff matrix $[1-c / 2,1-c, 1,0]$, for various sensitivities. For comparison we also add the plot of the mixed Nash equilibrium value (which corresponds formally to $s=\infty$ ) for the same game. All the plots intersect at the point $\left(c, p^{*}\right)=(2 / 3,1 / 2)$, since for $c=2 / 3$ the ME value $z^{*}=1$ (corresponding to $\left.p^{*}=1 / 2\right)$ is the zero of $W(z)$ in $(7)$ for all $s \geq 0$. For $c<2 / 3 p^{*}$ increases with $s$, for $c>2 / 3 p^{*}$ decreases with $s$, and is bigger than the Nash equilibrium value. For $s \rightarrow \infty p^{*}$ tends to the Nash equilibrium $p_{N}=2(1-c) /(2-c)$, cf. Lemma 3 in Appendix B.

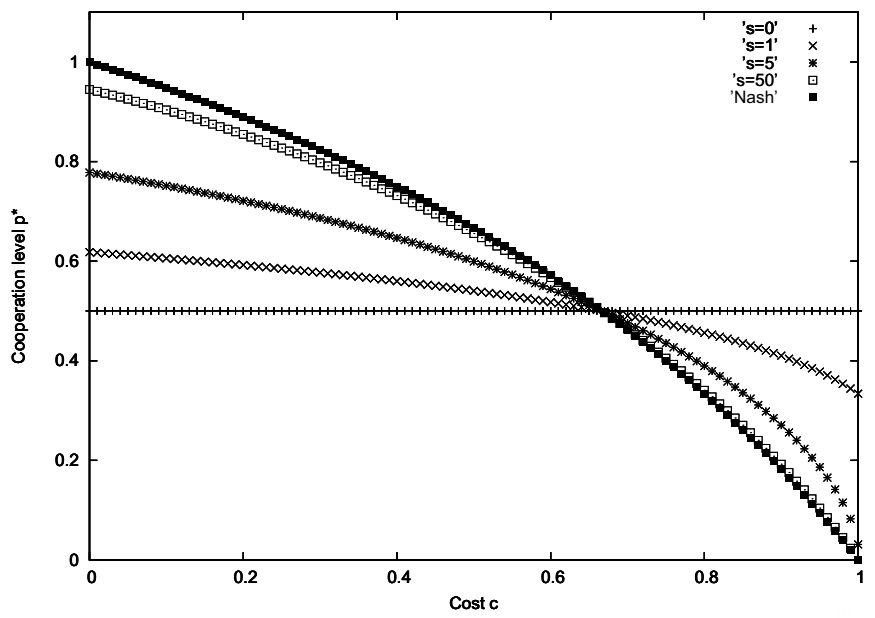

FIG. 3: Cooperation level vs. cost $c$ for the SD game with the normalized $(b=1)$ matrix $[1-c / 2,1-c, 1,0]$ for various sensitivities $s$. The 'Nash' curve corresponds to infinite sensitivity.

In Fig. 4 we plot the dependence of $p^{*}$ on the type of the game, for the sensitivities in the interval [0,10], and different payoffs $S$. The payoff matrix is $[3, S, 5,1]$. For $1>S \geq 0$ it describes the PD games, for $S=1$ the 'Weak' PD game, and for the payoff $S>1$ the Anti-coordination games (14). For all three types of the games, for each sensitivity $s>0$ the increase of the payoff $S$ implies the increase of $p^{*}$. Note that for $S>0$ the ME is unique. For $S=0$ there exists

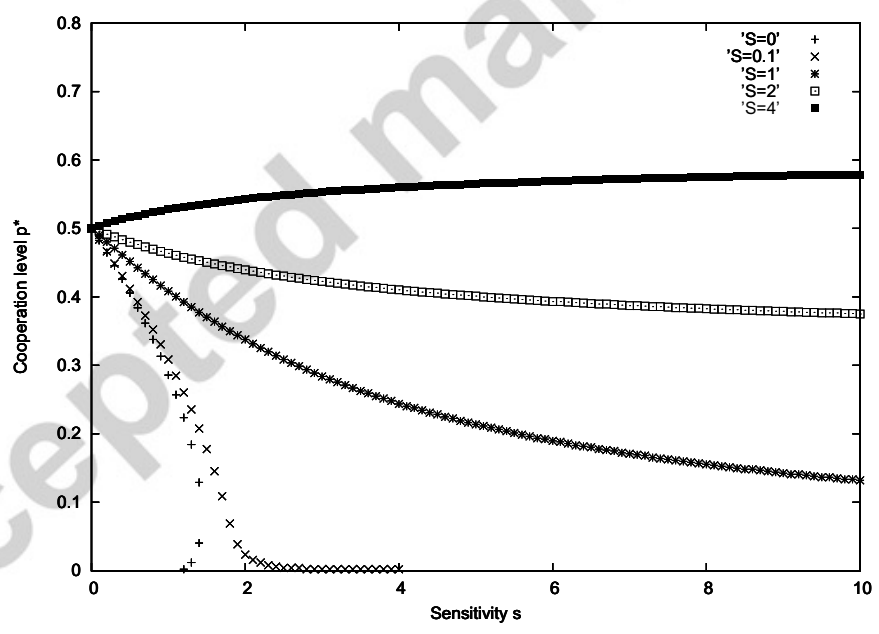

FIG. 4: Cooperation level vs. sensitivity for the payoff matrix $[3, S, 5,1]$ for different values of the sucker payoff S.

a second, unstable ME in an interval of $s$ around $s=1.4$, and there are no ME for larger sensitivities $s$, cf. Theorem 2, Appendix B. 
We consider the Coordination games defined by the payoff matrix:

$$
[R, S, T, P], \quad R>T, \quad P>S .
$$

The Nash equlibrium $p_{N}=(P-S) /(R-T+P-S)$ is unstable under the replicator dynamics. For example the Stag Hunt game (with $T \geq P$, and the first, C strategy corresponding to "Hunt Stag", the second, D - to "Hunt Hare") belongs to this class. In our dynamics, unlike the Anti-coordination games (14), the Coordination games can have two stable ME, as will be documented below. First we prove another uniqueness result.

As already stated, p. II.2 of Theorem 1 implies that for all 2-person games the condition $s \leq 1$ is sufficient for the uniqueness of the ME. For the Coordination games (16) we have a stronger uniqueness result:

\section{Lemma 2}

For the Coordination game (16) with $\epsilon:=\frac{S T}{R P}<1$ the $M E$ is unique for $s \leq \frac{1+\epsilon}{1-\epsilon}$.

Proof: The above inequality for $s$ is equivalent to $B>0$, i.e. to the sufficient condition II.1 of Theorem I for the uniqueness.

Below we study the Coordination games with the payoff matrix

$$
[R, S, S, R], R \neq S, R>S>0
$$

By inspection of (7), written for (17), we note that for all $0 \leq s<\infty$ there exists the ME $z^{*}=1$, i.e. $p^{*}=1 / 2$. The uniqueness depends on the payoff ratio $f:=R / S$ and on the sensitivity $s$. In Appendix B, Example 4 we formulate sufficient conditions for the existence of three ME for $s=2$.

\section{Example 1}

Fig. 5 shows the bifurcation of the stable equilibrium into three equilibria for two Coordination games. For the payoff matrix $[2,1,1,2]$ the equilibrium $p^{*}=0.5$ is globally stable and unique for $s \leq 3$ (in notation of Lemma $2 \epsilon=0.5$ ). The branching point $s=3$ corresponds to $z^{*}=1$, which is the third order zero of the function $W(z)$ in $(7)$. For $s>3$ the emerging two branches correspond to stable ME: for $s=3+\delta, \delta>0$ we calculate, from Theorem 1: $\Delta=6 \delta+0\left(\delta^{2}\right)>0, \quad B=-2-\delta<0$, and $U\left(z_{1}\right) U\left(z_{2}\right)<0$, which guarantees the existence of three ME.

The second game, with the payoff matrix $[2.01,1,1,2]$, indicates the sensitivity of the branching point to small perturbations of the payoff $R=2$. For $s<3.1$ the ME is unique. For $s \approx 3.1$ two new equilibria emerge, the middle one unstable, the lower stable, cf. the lower diagram of Fig. 5. For $s \rightarrow \infty$ two stable ME tend, as expected, to the replicator dynamics values $p^{*}=0, p^{*}=1$, the third ME tends to the unstable Nash equilibrium, as expected for the replicator dynamics for this coordination game.

\section{Example 2}

For the Stag Hunt game with the payoff matrix $[R, S, T, P]=[3,1,2,2]$ (and for all the games with $R+S=T+P$ ), the value $p^{*}=0.5$, corresponding to $z^{*}=1$, is the cooperation level for all sensitivities $s \geq 0$, since in this case $W\left(z^{*}=1\right)=0 \forall s \geq 0$. For $s \approx 3.8$ there emerge two additional ME, cf. Fig. 6 . For slightly larger $s$ the bigger ME tends to $p=0.5$, and merges with $p=0.5$ for $s=4$. For $s>4$ the ME $p^{*}=0.5$ is unstable, the other two branches are stable. As in the previous example, for $s \rightarrow \infty$ two stable ME tend to $p^{*}=0, p^{*}=1$, the third ME corresponds to the unstable Nash equilibrium with $p^{*}=0.5$.

\section{DISCUSSION}

We presented a model of population with complex personality profiles, and its applications to symmetric two-persons games, in particular those representing most important social dilemmas. The results are proved for well mixed infinite populations with random pairwise matching. The mechanism of the strategy updating based on imitation of more successful and more popular strategies solved the dilemma of the PD game.

We found stable equilibria of the considered dynamics, not present in the classical evolutionary game theory approach based on the replicator dynamics. In particular there exists a stable mixed equilibrium for the general PD game with arbitrary positive payoffs. Moreover, our theory reveals a 'subtle' structure of the asymptotic stationary solutions of the PD game under considered dynamics. In particular there are classes of the PD games with three stationary solutions, two of them locally stable for some intervals of the sensitivity parameter. The sensitivity parameter has a direct interpretation in the matching law of the operand-response theory. Similar structure can occur in the Coordination games, whereas for the Anti-coordination games (in particular for the SD game) the mixed equilibrium is unique and globally stable. 

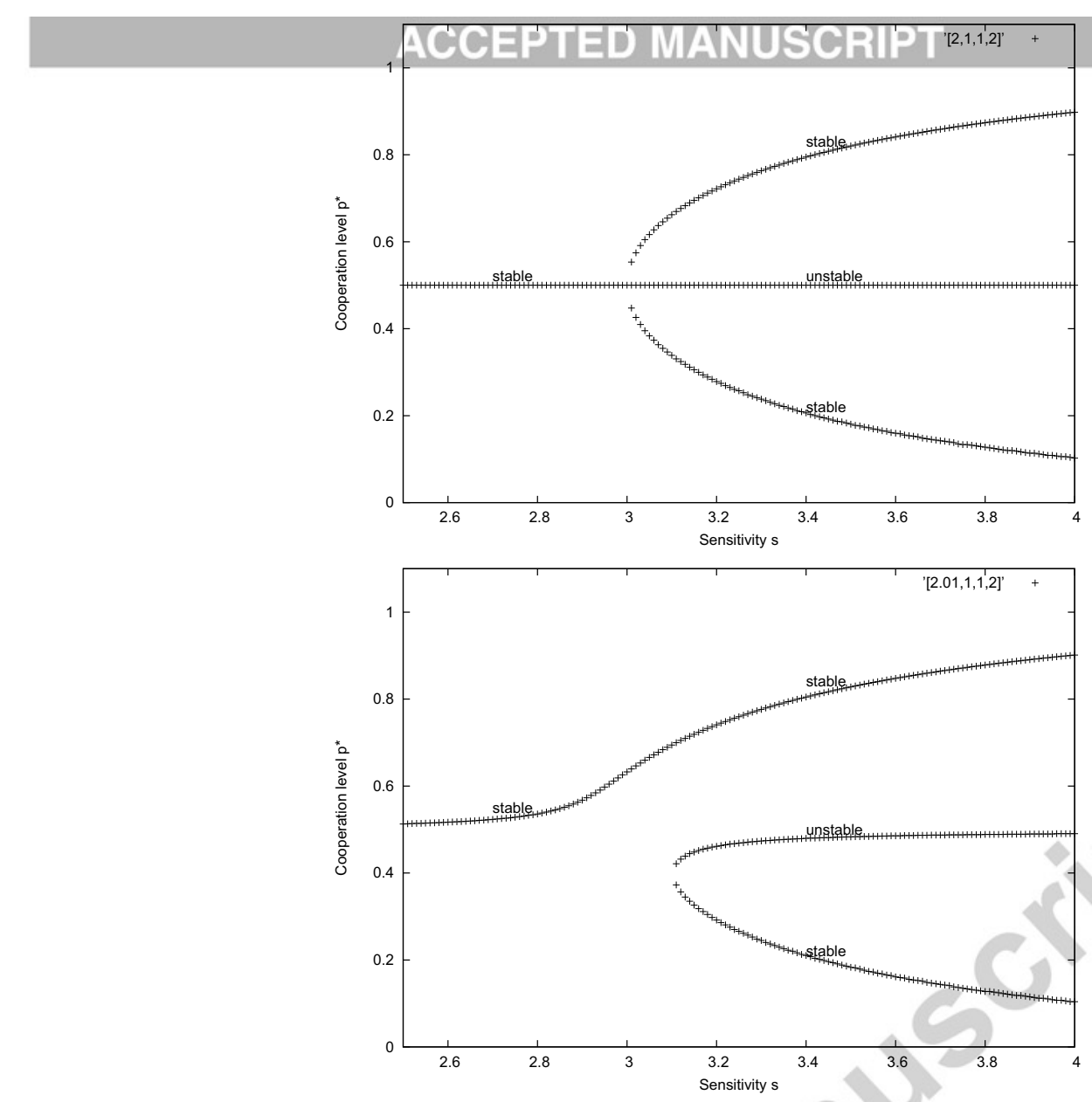

FIG. 5: Bifurcation of the unique ME into three ME for two Coordination Games with the payoff matrices [2,1,1,2], and $[2.01,1,1,2]$

The idea of combining together the bias towards imitating the strategies of the most payoff-successful agents and the strategies of the majority is not new, cf. e.g. Henrich and Boyd (2001) where it led to stabilization of the cooperation in populations. Multiple polymorphisms for the games with two strategies played in infinite populations have been found e.g. in the models of social dilemmas with synergy and discounting, cf. Hauert et al. (2006), in the aspiration-based models, cf. e.g. Palomino, Vega-Redondo (1999), Platkowski, Bujnowski (2009), in the Stag-Hunt N-person games, cf. Pacheco et al. (2009), in the N-person Snowdrift games, cf. Souza et al. (2009). In the presented imitation model, in which the "sociological" aspect of the attractiveness of the strategies is taken into account through its dependence on the actual propensities of the strategies, the multiple polymorphisms can exist already in the two-person games.

Further research will be focused on generalization for systems with more than two behavioral types, non-symmetric games, other attractiveness functions, and more general psychological types. Preliminary investigations indicate interesting dynamics in such more complicated situations. It will also be interesting to allow the actors to change their personalities during the interactions.

Acknowledgements. TP was supported by the Polish Government Grant no. N201 362536. The author is grateful to two anonymous referees for helpful comments.

[1] Aviles, N., 1999. Cooperation and non-linear dynamics: An ecological perspective on the evolution of sociality. Evol. Ecol. Res. 1, 459-477.

[2] Axelrod R., 1984. The evolution of cooperation. New York: Basic Books.

[3] Cobb, C.W., Douglas, P.H., 1928. A Theory of Production. American Economic Review 18 (Supplement) 139-165. 


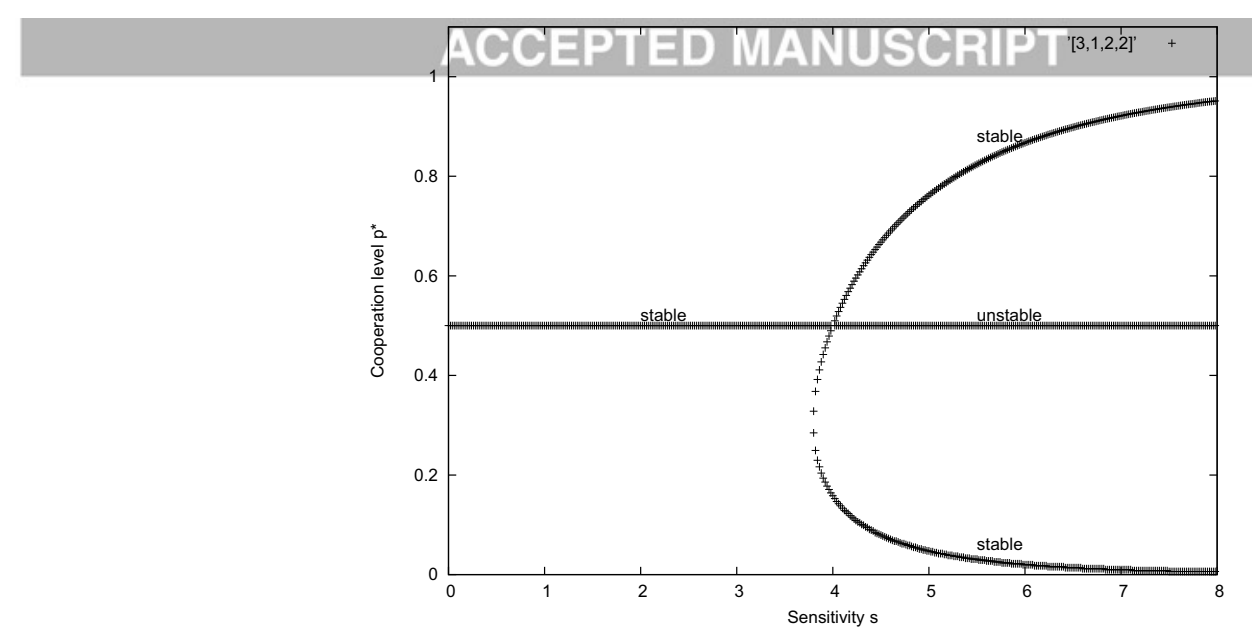

FIG. 6: Bifurcation of the unique ME into three ME for the Stag Hunt Game with the payoff matrix [3, 1, 2, 2].

[4] Gintis, H., 2009. The Bounds of Reason. Game Theory and the Unification of the Behavioral Sciences. Princeton University Press. Princeton and Oxford.

[5] Hauert, C., Doebeli, M., 2004. Spatial structure often inhibits the evolution of cooperation in the snowdrift game. Nature $428,643-646$.

[6] Hauert, C., Michor, F., Nowak, M.A., and Doebeli, M., 2006. Synergy and discounting of cooperation in social dilemmas. J. Theor. Biol. 239, 195-202.

[7] Henrich, J., Boyd, R., 2001. Why People Punish Defectors. Wak Conformist Transmission can Stabilize Costly Enforcement of Norms in Cooperative Dilemmas. J. Theor. Biol. 208, 79-89.

[8] Herrnstein, R.J., 1997. The Matching Law. New York: Harvard University Press.

[9] McElreath, R., Boyd, R., 2007. Mathematical Models of Social Evolution: A Guide for the Perplexed. The University of Chicago Press. Chicago and London.

[10] Nowak, M.A., 2006. Evolutionary Dynamics: Exploring the Equations of Life. The Belkinnap Press of Harvard University Press. Cambridge, Massachusetts and London.

[11] Pacheco, J.M., Santos, F., C., Souza, M., O., and Skyrms, B. 2009. Evolutionary dynamics of collective action in N-person stag hunt dilemmas. Proc. R. Soc. B 276, 315-321.

[12] Palomino, F.,Vega-Redondo, F., 1999. Convergence of aspirations and (partial) cooperation in the prisoner's dilemma. Int. J. Game Theory 28, 465-488

[13] Platkowski, T., Poleszczuk, J., 2009. Operant Response Theory of Social Interactions. eJourn. Biol. Sci. 1, 1, 1-10.

[14] Platkowski, T., Bujnowski, P., 2009. Cooperation in aspiration-based N-person prisoners dilemmas. Phys. Rev. E 79, 036103

[15] Seth, A. K., 2002. Evolving Behavioral Choice: An Investigation into Herrnsteins Matching Law.

[16] Souza, M. O., Pacheco, J., M., Santos, F., C., 2009. Evolution of cooperation under N-person snowdrift games. J. Theor. Biol. 260(4), 581-588.

[17] Sugden, R.,1986. The Economics of Rights, Co-operation and Welfare. Blackwell, Oxford, New York.

[18] Szabo, G., Fath, G., 2007. Evolutionary games on graphs. Physics Reports 446 (4-6), 97-216

[19] Vega-Redondo, F., 2003. Economics and the theory of games. Cambridge, New York: Cambridge University Press

[20] Weibull, J. W., 1995. Evolutionary Game Theory. Cambridge: The MIT Press.

[21] Weber, M., 1968. Economy and Society. New York. Bedmister Press.

[22] Traulsen A., Shoresh N., Nowak M.A., 2008. Analytical Results for Individual and Group Selection of Any Intensity. Bull. Math. Biol. 70, 1410-1424.

\section{Proposition 1}

$p^{*} \in(0,1)$ is the stationary solution of (5) iff $z^{*}=p^{*} /\left(1-p^{*}\right)$ is the stationary solution of of (7); $p^{*}$ is stable (unstable) iff $z^{*}$ is stable (unstable).

\section{Proof}

The proof follows from the fact that omitting the positive multiplicative terms on the rhs of eq. (5) we do not change its equilibria and their stability.

After substituting $z=p_{1} /\left(p_{1}-1\right)$ eq. (5) takes the form

$$
\left.\dot{z}=P(z)\left[\left(R(z)^{s}\right)\right)^{\alpha}-z^{\alpha}\right],
$$




\section{Proof of Theorem 1, p. II-IV:}

II. We calculate $U^{\prime}(z)=W_{1}(z) \tilde{P}(z)$, where $\tilde{P}(z)>0 \forall z>0$, and

$$
W_{1}(z)=z^{2}+B z+\frac{S P}{R T} .
$$

Thus, extrema of $U$ are zeros of the polynomial $W_{1}$. II.1 follows from the fact that:

for all $z>0$ and $\Delta<0 W_{1}(z)>0$;

for $\Delta=0 \exists ! z^{*}: W_{1}\left(z^{*}\right)=0$, i.e. $U^{\prime}\left(z^{*}\right)=0: z^{*}=-B / 2$, thus can not be an extremal point.

II.2 follows from the following observations:

for $B=0 W_{1}>0 \forall z \geq 0$, therefore $U^{\prime}(z)>0 \forall z \geq 0, U$ is strictly monotonic;

for $B>0$ : if $\Delta \geq 0$ then $z_{1}<0$ and $z_{2}<0$, where $W_{1}\left(z_{i}\right)=0, i=1,2$, therefore $U(z)$ has no extrema for $z>0$.

if $\Delta<0$ then $W_{1}(z)>0 \forall z \geq 0$.

II.3 follows by noting that $\forall s \geq 0 B>0$, and applying II.2.

III follows from the observation that the graph of $U(z)$ has at most three common points with the z-axis.

IV: $<=$ : For $\Delta>0$ both roots $0<z_{1}<z_{2}$ of $W_{1}$ are positive. The condition $U\left(z_{1}\right) U\left(z_{2}\right)<0$ together with the limit values of $U(z)$ imply $U\left(z_{1}\right)>0$ and $U\left(z_{2}\right)<0$. In consequence the graph of $U(z)$ crosses the positive part of the $z$ axis three times.

$=>$ : The existence of three ME, i.e. that $U$ crosses the $z$-axis three times, implies that $U(z)$ must have two extrema, say at $\bar{z}_{1}>0$ and $\bar{z}_{2}>0$, i.e. $W_{1}\left(\bar{z}_{1}\right)=W_{1}\left(\bar{z}_{2}\right)=0$. Thus, $\Delta>0$, and the relation $\bar{z}_{1}+\bar{z}_{2}=-B>0$ implies $B<0$. The condition that $U(z)$ crosses the z-axis three times implies $U\left(\bar{z}_{1}\right)>0$ and $U\left(\bar{z}_{2}\right)<0$.

\section{Remarks}

The uniqueness condition II.3 can be given the following interpretation. Each 2-person symmetric game can be considered as the sum of two basic games: the pure Coordination game (CG) with the payoff matrix $[R, 0,0, P]$, and pure Anti - coordination game (AG) with the payoff matrix $[0, S, T, 0]$. Then II.3 states that the ME is unique if the product of the positive payoffs in AG is bigger than that in CG.

We note that (5) is invariant in $[0,1]$, that for $\alpha=\beta=1 z^{*}=1$ is the unique, globally stable ME. Two ME are also possible for particular cases in which the graph of $\mathrm{U}$ is tangent to the $\mathrm{z}$ axis. Such situations are "unstable" with respect to arbitrarily small perturbations of the relevant parameters of the model, and are not considered here.

\section{APPENDIX B}

For convenience of the reader we formulate the existence theorem for the matrices with nonnegative entries. The proofs are similar to the proofs of the existence theorems considered above and will be omitted here.

For $S=0$ and $R, T, P>0$, e.g. for the PD matrix corresponding to the Axelrod's tournament, Axelrod (1984), the existence of ME is not guaranteed, cf. Platkowski and Poleszczuk (2009).

\section{Theorem 2}

For the payoff matrix $[R, 0, T, P], R>0, T>0, P>0$ :

For $s<1$ there exists an unique $M E$, i.e. positive solution of eq. (7).

For $s>1$ the existence and uniqueness of $M E$ depend on the function

$$
G(s)=\frac{1}{P}\left(\frac{s-1}{T}\right)^{s-1}\left(\frac{R}{s}\right)^{s}:
$$

If $G(s)>1$ then there exist two $M E$. If $G(s)=1$ then there exists an unique $M E z=(s-1) P / T$. If $G(s)<1$ then there are no $M E$.

For $s=1$ and the payoff matrix $[R, S, T, P], T>0$ :

(i) for $S>0$ there exists exactly one, unique $M E$

(ii) for $S=0$ : if $R>P$ then the $M E$ is unique; if $R \leq P$ then there are no $M E$.

\section{Lemma 3: ME for the Snow-Drift Game}

Consider the SD game $[1-c / 2,1-c, 1,0], 0<c<1$ and $s=1$. For $c>2 / 3$ we have $p^{*}>p_{N}$, for $c<2 / 3 p^{*}<p_{N}$, and for $c=2 / 3 p^{*}=p_{N}$. 
Proof. The Nash equilibrium is $p_{N_{\Lambda}}=2(1-c) /(2-c)$. For $s=1-W(z)=z^{2}-(1-c / 2) z-(1-c)$. Denote $z(c)-$ the positive solution of eq. $W(z)=0, p^{*}=z(c) /(z(c)+1)$. The condition $p^{*}>p_{N}$ is equivalent to $c^{2}-10 c+8<0$, satisfied for $1>c>2 / 3$.

For $s \neq 1$ we checked numerically, for $c>2 / 3$, the validity of the inequality $p^{*} \equiv z^{*} /\left(z^{*}+1\right)>2(1-c) /(2-c)=p_{N}$, $z^{*}=z^{*}(c)$ being the solution of $W(z)=z^{1+1 / s}-(1-c / 2) z-(1-c)=0$, cf. Fig. 3 .

\section{Example 3}

For the Coordination game $[4,1,2,3] z^{*}=1$ is ME for all $s \geq 0$. The condition II.2 of Theorem 1 is equivalent to $s \leq 7 / 5$. The sufficient conditions II.1,2 give a stronger estimate: $s_{1} \leq s \leq s_{2}$, where $s_{i}=\frac{7 \mp \sqrt{6}}{5}, i=1,2$. Thus, for $s \in\left[0, s_{2}\right]$, where $s_{2} \approx 2.4 z^{*}=1$ is the unique ME.

For $s>s_{2}$ two new ME $z_{1}<z_{2}$ emerge, as can be verified by checking the sufficient conditions IV of Theorem 1 . For $s=2.5 z_{2}=1$ is neutrally stable, $z_{1}$ is globally attractive (except $z_{2}$ ). For $s>2.5 z_{2}$ bifurcates into two ME: the unstable $z_{2}=1$, and the stable $z_{3}>1$. For $s \rightarrow \infty$ we recover two stable Nash equilibria $p^{*}=0$ and $p^{*}=1$, and the unstable one $p^{*}=0.5$.

\section{Example 4}

For the Coordination games (17) with $s=2$ and $f=\frac{R}{S} \leq 1+\sqrt{2}$ the ME $p^{*}=0.5$ is unique. For $f>1+\sqrt{2}$ there are three ME: $p^{*}=0.5, p_{1}^{*}<0.5, p_{2}^{*}>0.5$. For $f \rightarrow \infty p_{1}^{*} \rightarrow 0, p_{2}^{*} \rightarrow 1$.

Proof: For $s=2$ the equilibrium condition $W(z)=0$, cf.(7), takes the form

$$
(z-1)\left[z^{2}+\left(1+2 f-f^{2}\right) z+1\right]=0 .
$$

The result follows from the analysis of zeros of the binomial $z^{2}+\left(1+2 f-f^{2}\right) z+1$. 